
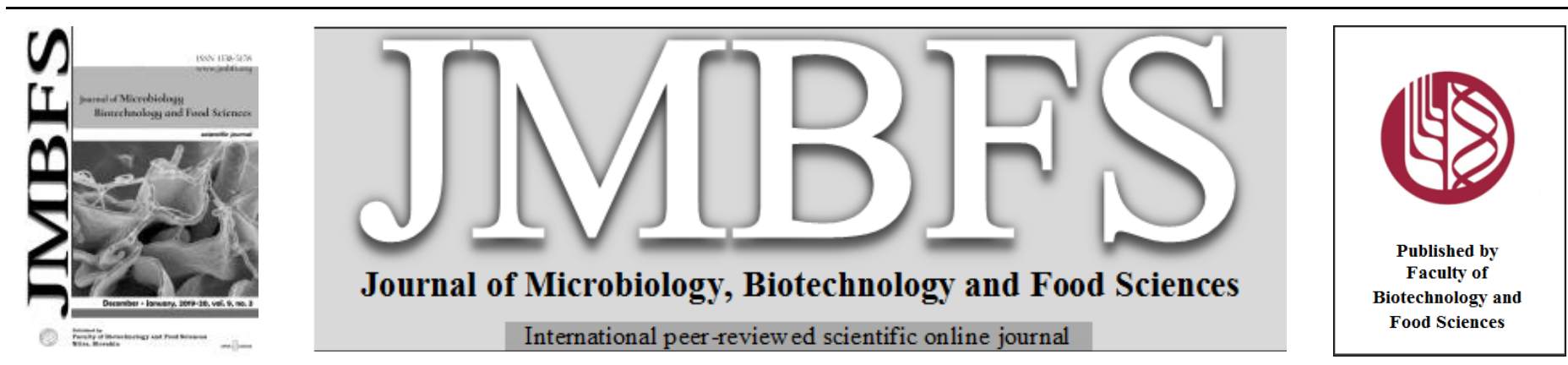

\title{
BIOCHEMICAL CHARACTERIZATION AND ANTIMICROBIAL SUSCEPTIBILITY OF Bacillus cereus ISOLATES FROM SOME RETAILED FOODS IN OGUN STATE, NIGERIA
}

\author{
Titilayo O. Adesetan, ${ }^{1,2}$ Prof. Moses O. Efuntoye ${ }^{1}$ and Prof. Olubukola O. Babalola ${ }^{2 *}$
}

Address(es): Prof. Olubukola O. Babalola,

${ }^{1}$ Department of Microbiology, Olabisi Onabanjo University, Ogun State, PMB 2002, Ago - Iwoye, Nigeria.

${ }^{2}$ Food Security and Safety Niche, Faculty of Natural and Agricultural Sciences, North-West University, Mafikeng Campus, Private Bag X2046, Mmabatho 2735, South Africa.

*Corresponding author: olubukola.babalola@nwu.ac.za

doi: 10.15414/jmbfs.2019/20.9.3.616-621

ARTICLE INFO

Received 20. 9. 2020

Revised 13. 6. 2019

Accepted 13. 6. 2019

Published 1. 12. 2019

Regular article open $\partial_{\text {ACCESS }}$

\begin{abstract}
Food borne disease caused by Bacillus cereus has been a major health issue because of its ability to cause two syndromes: diarrhoeal and emetic which sometimes lead to death. Six hundred (600) samples of some retailed foods: cooked rice, jollof rice, fried rice, meat pie, smoked fish: African chad, Titus, blue whiting, fried meat, smoked hide, carrot, runner beans, cabbage and raw green pea were collected from the eight main markets in Ogun State. Serial dilutions of the samples were carried out and cultured on Mannitol Egg Yolk Polymyxin Agar (MYP) using the spread plate technique. B. cereus was confirmed with standard biochemical methods. Antibiotic susceptibility was performed by the Kirby - Bauer disc diffusion method with nine antibiotics. The mean microbial load in the retailed food was in the range of $1.00 \times 10^{4}-8.92 \times 10^{4} \mathrm{cfu} / \mathrm{g}$. All the isolates were gram positive rods, catalase and citrate positive. Most of the isolates were motile $(97.7 \%)$. Two hundred and twenty one (221) isolates were confirmed as B. cereus with biochemical tests. They were $100 \%$ sensitive to gentamicin and $100 \%$ resistant to penicillin and ampicillin which are $\beta$ - lactam antibiotics. All the isolates showed resistance to more than two antibiotics. This study has clearly revealed the presence of $B$. cereus in some retailed foods sold in Ogun State, Nigeria in which all the isolates were resistant to $\beta$-lactam antibiotics. Therefore, extreme caution should be taken when handling foods to avoid contamination by $B$. cereus and prevent any future food-borne outbreak by $B$. cereus.
\end{abstract}

Keywords: Antibiotics, Bacillus cereus, biochemical tests, Mannitol Egg Yolk Polymyxin Agar (MYP), retailed foods

\section{INTRODUCTION}

Food is essential for man's sustenance. Microorganisms attack our food for survival thereby causing deterioration and contamination of the food. Therefore, the food we eat can be beneficial to our body and at the same time cause harm when microorganisms in interaction with the food release their metabolites thereby causing illness. One of such bacteria is Bacillus cereus. Members in the $B$. cereus group are gram positive, ubiquitous rod shaped spore former. They are known to survive virtually in all environments because of their ability to form spores that are resistant to heat and acid. There are seven members in the group namely: B. anthracis, $B$. cereus sensu stricto, $B$. thuringiensis, $B$. mycoides, $B$. pseudomycoides, $B$. weihenstephanensis and B. cytotoxicus. The first three are the most important within the group (Lapidus et al. 2008; Logan and de Vos, 2009).

The economic situation of some countries has encouraged the consumption of ready-to-eat foods and establishment of more fast foods restaurant, canteens and road side food outlets. These foods are believed to be affordable and easily accessible. Food-borne pathogens may multiply in foods that are not well handled, prepared or stored due to lack of hygiene and poor sanitation (Angelidis et al., 2006; Desai and Varadaraj, 2009).

B. cereus causes two types of poisonings namely: diarrhoeal with abdominal cramp and diarrhoea as the symptom and emetic with nausea and vomiting. Diarrhoeal poisoning is associated with proteins such as meat while starchy foods such as rice and pasta have been linked to the emetic poisoning. It had been reported to cause illnesses (Ghelardi et al., 2002; Martinelli et al., 2013; Zhou et al., 2014) or death (Dierick et al., 2005; Naranjo et al., 2011).

$B$. cereus can survive an array of stress situations including those found in some foods because of its ability to form spores. It has been isolated from a wide variety of foods, including milk and dairy products (De Jonghe et al., 2008; Arslan et al., 2014), rice and pasta (Rajkovic et al., 2013), infant foods (Organji et al., 2015), spices (Iurlina et al., 2006), meat product (Gueven et al., 2006), seafood (Das et al., 2009), fresh vegetables (Valero et al., 2002), vegetable salad (Valero et al., 2007) and ready to eat foods (Fang $\boldsymbol{e t}$ al., 2003;
Samapundo et al., 2011). Bacillus infections can be treated with antibiotics such as vancomycin, clindamycin, ciprofloxacin, and gentamicin. Penicillins and ampicillins are not effective (Lindback and Granum, 2006; Chon et al., 2012) because they produce $\beta$-lactamase that hydrolyses the $\beta$-lactam ring of the antibiotics.

Review of literatures in Nigeria showed that there is scarcity of information on characterization of B. cereus in retailed foods in Ogun State; therefore, there is need to characterize these food pathogens using both morphological and biochemical properties and determine their susceptibility to common antibiotics to forestall any future occurrence of food borne outbreak.

\section{MATERIALS AND METHODS}

\section{Sample Collection}

Six hundred (600) samples of ready-to-eat foods and vegetables such as cooked white rice (Oryza sativa), jollof rice, fried rice, meat pie, cooked spaghetti, smoked African chad (Etmalosa fimbriata), smoked Titus (Scumber scumbrus), smoked blue whiting (Micromesistius poutassou), smoked hide, fried meat (Bos taurus), green pea (Pisum sativum), sweet pepper (Capsicum annuum), runner bean (Phaseolus vulgaris), cabbage (Brassica oleracea) and carrot (Daucus carrota) (Table 1) were collected randomly inside sterile sampling bags/plastics from food outlets/canteens in the markets namely: Oke-Aje and Ago-Iwoye markets (Ijebu), Sagamu and Ikenne markets (Remo), Kuto and Omida markets (Egba) and Ayetoro and Imeko markets (Yewa) in the four geographical regions in Ogun State, they were sealed to prevent contamination and transported to the laboratory for analysis. Forty samples were collected from each food type. The samples were collected in the morning and it was ensured that the vegetables were fresh ones without any sign of deterioration. The samples were collected between September 2013 and April 2015. The analyses were carried out at the Laboratories of the Department of Microbiology, Olabisi Onabanjo University, Ago - Iwoye, Nigeria and Microbial Biotechnology Laboratory of North - West University, Mafikeng Campus, South Africa. 
Table 1 Number of food samples collected from each division

\begin{tabular}{llcccccc}
\hline Food groups & Food name & Food Code & Egba & Ijebu & Remo & Yewa & Total \\
\hline Starch & Cooked Rice & WR & 10 & 10 & 10 & 10 & 40 \\
& Jollof Rice & JR & 10 & 10 & 10 & 10 & 40 \\
& Fried Rice & FR & 10 & 11 & 11 & 8 & 40 \\
& Meat pie & MP & 10 & 10 & 10 & 10 & 40 \\
& Spaghetti & SG & 10 & 10 & 10 & 10 & 40 \\
Protein & Fried Meat & MT & 10 & 10 & 10 & 10 & 40 \\
& Smoked Titus & TT & 10 & 10 & 10 & 10 & 40 \\
& Smoked African chad & SW & 10 & 10 & 10 & 10 & 40 \\
& Smoked Blue whiting & PN & 10 & 10 & 10 & 10 & 40 \\
& Smoked Hide & PM & 10 & 10 & 10 & 10 & 40 \\
Vegetable & GP & 12 & 12 & 12 & 4 & 40 \\
& Green pea & SP & 10 & 10 & 10 & 10 & 40 \\
& Sweet pepper & CB & 11 & 12 & 11 & 10 & 40 \\
& Cabbage & CR & 10 & 10 & 10 & 10 & 40 \\
& Carrot & RB & 10 & 10 & 10 & 10 & 40 \\
& Runner Bean & & 153 & 155 & 154 & 138 & 600 \\
\hline
\end{tabular}

\section{Sample preparation}

Samples of meat, runner beans, carrot, cabbage, smoked hide and sweet pepper were chopped into pieces with sterile knife after which $10 \mathrm{~g}$ of each sample was added to $100 \mathrm{ml}$ sterile peptone water (1:10 dilution) and diluted up to $10^{-5}$.

\section{Isolation of $B$. cereus}

Mannitol Egg Yolk Polymyxin (MYP) agar (Oxoid, UK) plates were inoculated with $0.1 \mathrm{ml}$ of appropriate dilution and spread evenly onto surface of each plate with sterile bent glass rod. Plates were incubated at $30^{\circ} \mathrm{C}$ for $18 \mathrm{hrs}$ and observed for colonies surrounded by precipitate zone, which indicated that lecithinase was produced (Tallent et al., 2001).

\section{Microbial load}

After incubating for $24 \mathrm{hrs}$, the plates with distinct colonies were counted and then multiplied with the dilution factor to get the total bacteria count in colony forming unit per gram (cfu/g)

\section{Confirmation of B. cereus}

The colonial morphology of the isolates was first recorded after which the biochemical tests based on Food and Drug Administration (FDA) methods as described by Tallent et al. (2001) and Cheesebrough (2000) were carried out Briefly, five (5) or more eosin pink, lecithinase-positive colonies from MYP agar (Oxoid, UK) plates were transferred to nutrient agar plates (MAST, Merseyside $\mathrm{UK}$ ) and incubated for $24 \mathrm{~h}$ at $30^{\circ} \mathrm{C}$. The isolates were gram stained and confirmed using the following tests: glucose fermentation, nitrate reduction, motility test, catalase test, citrate utilization, starch hydrolysis, casein hydrolysis, rhizoid growth, haemolysis and growth at $42^{\circ} \mathrm{C}$.

\section{Antimicrobial susceptibility test}

This test was performed using Kirby-Bauer Disc Diffusion method (Bauer et al 1966). Nine (9) different antibiotics (Bio-Rad, USA) were employed: penicillin (PEN) 10IU, ciprofloxacin (CIP) $5 \mu \mathrm{g}$, tetracycline (TET) $30 \mu \mathrm{g}$, erythromycin (ERY) $15 \mu \mathrm{g}$, gentamicin (GMN) $10 \mu \mathrm{g}$, amoxicillin-clavulanic acid (AMC) 30 $\mu \mathrm{g}$, vancomycin (VAN) $30 \mu \mathrm{g}$, ampicillin (AMP) $10 \mu \mathrm{g}$ and clindamycin (CMN) $2 \mu \mathrm{g}$ (Oxoid, UK). The results were interpreted using Clinical Laboratory Standard Institute CLSI (2013) guideline for Gram positive and /or aerobic bacteria.

\section{Statistical Analysis}

Data were analyzed using the Statistical Package for Social Sciences (SPSS) version 20.0 (IBM Corp, 2011). Mean values were compared using Analysis of Variance (ANOVA). Results were presented as Mean \pm Standard deviation. Post hoc test was done using the Student-Newman-Keuls (SNK). p $<0.05$ was considered to be statistically significant.

\section{RESULTS}

\section{Microbial load of retailed foods}

The microbial load in the food samples is presented on Table 2. Food with the highest microbial load was spaghetti from Yewa with mean bacterial load of 8.92 $\mathrm{x} 10^{4} \mathrm{cfu} / \mathrm{g}$, followed by White rice from Yewa with $4.18 \times 10^{4} \mathrm{cfu} / \mathrm{g}$ while the food with the least count was green pea from Ijebu -Ode with mean bacterial load of $1.00 \times 10^{4} \mathrm{cfu} / \mathrm{g}$. The One Way Analysis of Variance (ANOVA) revealed a significant difference ( $p \leq 0.05$ in the microbial load of the food samples SP and SW collected from all the locations. There was also a significant difference $(\mathrm{p} \leq 0.05)$ between the locations where the samples were collected.

Table 2 Mean Microbial load in food samples collected from different divisions (x10 $\mathrm{cfu} / \mathrm{g})$

\begin{tabular}{lcccc}
\hline $\begin{array}{l}\text { Location / } \\
\text { Food code }\end{array}$ & Egba & Ijebu & Remo & Yewa \\
\hline WR & $3.42 \pm 1.22^{\mathrm{a}}$ & $2.82 \pm 0.83^{\mathrm{a}}$ & $3.92 \pm 0.76^{\mathrm{a}}$ & $4.18 \pm 0.80^{\mathrm{a}}$ \\
JR & $3.70 \pm 0.20^{\mathrm{a}}$ & $3.00 \pm 0.57^{\mathrm{a}}$ & $4.90 \pm 0.10^{\mathrm{a}}$ & $0.00 \pm 0.00^{\mathrm{b}}$ \\
FR & $3.10 \pm 1.27^{\mathrm{b}}$ & $6.85 \pm 0.64^{\mathrm{a}}$ & $1.20 \pm 0.20^{\mathrm{c}}$ & $3.10 \pm 0.10^{\mathrm{b}}$ \\
MP & $1.90 \pm 0.28^{\mathrm{a}}$ & $2.03 \pm 1.33^{\mathrm{a}}$ & $1.60 \pm 0.14^{\mathrm{a}}$ & $1.60 \pm 0.56^{\mathrm{a}}$ \\
SG & $5.28 \pm 3.18^{\mathrm{a}}$ & $5.68 \pm 4.17^{\mathrm{a}}$ & $4.08 \pm 1.81^{\mathrm{a}}$ & $8.92 \pm 2.60^{\mathrm{a}}$ \\
MT & $2.78 \pm 1.59^{\mathrm{a}}$ & $2.00 \pm 0.72^{\mathrm{a}}$ & $2.70 \pm 1.44^{\mathrm{a}}$ & $2.62 \pm 0.60^{\mathrm{a}}$ \\
TT & $3.48 \pm 0.94^{\mathrm{a}}$ & $3.64 \pm 1.55^{\mathrm{a}}$ & $3.00 \pm 0.96^{\mathrm{a}}$ & $5.23 \pm 0.42^{\mathrm{a}}$ \\
SW & $6.03 \pm 3.46^{\mathrm{a}}$ & $2.86 \pm 1.49^{\mathrm{b}}$ & $2.38 \pm 0.54^{\mathrm{b}}$ & $4.70 \pm 1.04^{\mathrm{a}}$ \\
PN & $3.65 \pm 4.31^{\mathrm{b}}$ & $2.90 \pm 0.28^{\mathrm{b}}$ & $6.50 \pm 0.50^{\mathrm{a}}$ & $3.10 \pm 2.12^{\mathrm{b}}$ \\
PM & $4.00 \pm 0.50^{\mathrm{a}}$ & $4.37 \pm 1.21^{\mathrm{a}}$ & $4.35 \pm 0.21^{\mathrm{a}}$ & $4.70 \pm 0.20^{\mathrm{a}}$ \\
GP & $1.35 \pm 0.21^{\mathrm{a}}$ & $1.00 \pm 0.50^{\mathrm{a}}$ & $1.38 \pm 0.28^{\mathrm{a}}$ & $2.00 \pm 0.60^{\mathrm{a}}$ \\
SP & $1.57 \pm 0.40^{\mathrm{b}}$ & $2.00 \pm 0.60^{\mathrm{b}}$ & $2.18 \pm 0.17^{\mathrm{b}}$ & $3.90 \pm 0.28^{\mathrm{a}}$ \\
RB & $4.04 \pm 0.57^{\mathrm{a}}$ & $4.10 \pm 0.96^{\mathrm{a}}$ & $2.88 \pm 1.04^{\mathrm{a}}$ & $3.96 \pm 1.68^{\mathrm{a}}$ \\
CB & $2.77 \pm 0.68^{\mathrm{b}}$ & $2.53 \pm 0.51^{\mathrm{b}}$ & $1.70 \pm 0.26^{\mathrm{b}}$ & $3.30 \pm 0.30^{\mathrm{a}}$ \\
CR & $2.00 \pm 0.60^{\mathrm{b}}$ & $3.70 \pm 0.20^{\mathrm{a}}$ & $2.65 \pm 0.92^{\mathrm{b}}$ & $3.40 \pm 0.40^{\mathrm{a}}$ \\
\hline abc & &
\end{tabular}

${ }^{a b c}$ Means ( \pm Standard deviation) in the same row having similar superscripts are not significantly different at $\mathrm{p}<0.05 . \mathrm{n}=181$

WR-White Rice JR-Jollof Rice FR-Fried Rice TT-Smoked Titus GP-Green Pea $\quad$ SG-Spaghetti $\quad$ RB - Runner Bean $\quad$ MP - Meat pie CR-Carrot $\quad$ MT-Meat $\quad$ CB - Cabbage $\quad$ SW - Smoked African chad $\quad$ SP-Sweet Pepper $\quad$ PN-Smoked blue whiting PM-Smoked hide

\section{Colonial morphology of $B$. cereus}

The isolates formed opaque, irregular, smooth/rough, creamish colonies on Nutrient Agar. Some also showed waxy growth while others had short hair-like growth around the colony. Most of the colonial characteristics exhibited were typical of Bacillus cereus.

\section{Gram stain reaction and biochemical characterization}

All the isolates were Gram positive rods. Many were in chains while few were single rods. Some have central to sub-terminal spores which do not swell the sporangium. All the isolates were catalase and citrate positive. $95.9 \%$ of the isolates were haemolytic, $97.7 \%$ were motile, $77.4 \%$ and $86.0 \%$ hydrolysed starch and casein respectively. They grew well at $42^{\circ} \mathrm{C}$ (Table 3 ). 
Table 3 Biochemical tests pattern for B. cereus isolates from some retailed foods

\begin{tabular}{|c|c|c|c|c|c|c|c|c|c|c|c|c|c|c|}
\hline 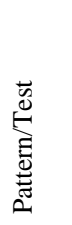 & 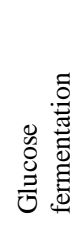 & 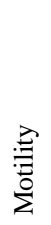 & 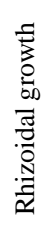 & 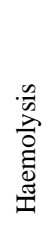 & 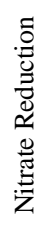 & 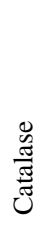 & 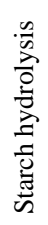 & 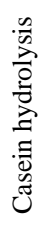 & 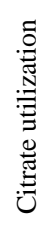 & 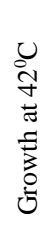 & $\begin{array}{l}\tilde{0} \\
\text { क्D } \\
\text { II }\end{array}$ & $\stackrel{\Xi}{0}$ & ڤ્ב & 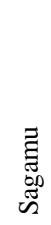 \\
\hline I & + & + & - & - & + & + & + & + & + & + & 1 & 0 & 0 & 1 \\
\hline II & + & + & - & $\beta$ & + & + & + & + & + & + & 32 & 41 & 40 & 31 \\
\hline III & + & + & - & $\alpha$ & + & + & - & + & + & + & 1 & 0 & 0 & 0 \\
\hline IV & + & + & - & $\beta$ & + & + & - & + & + & + & 9 & 6 & 7 & 13 \\
\hline V & + & + & - & $\alpha$ & + & + & - & - & + & + & 0 & 0 & 1 & 2 \\
\hline VI & + & + & - & $\beta$ & + & + & + & - & + & + & 7 & 5 & 3 & 7 \\
\hline VII & + & + & - & - & + & + & - & + & + & + & 1 & 1 & 1 & 1 \\
\hline VIII & + & + & - & - & + & + & + & - & + & + & 0 & 1 & 0 & 0 \\
\hline IX & + & - & - & - & + & + & + & - & + & + & 0 & 1 & 0 & 0 \\
\hline X & + & - & - & $\beta$ & + & + & - & + & + & + & 0 & 2 & 0 & 0 \\
\hline XI & + & + & - & - & + & + & - & - & + & + & 0 & 1 & 0 & 0 \\
\hline XII & + & + & - & $\alpha$ & + & + & + & + & + & + & 0 & 0 & 1 & 0 \\
\hline XIII & + & + & - & $\beta$ & + & + & - & - & + & + & 0 & 0 & 2 & 0 \\
\hline XIV & + & - & - & $\beta$ & + & + & + & + & + & + & 0 & 0 & 1 & 0 \\
\hline XV & + & - & - & $\alpha$ & + & + & - & - & + & + & $\begin{array}{l}1 \\
52\end{array}$ & $\begin{array}{l}0 \\
58\end{array}$ & $\begin{array}{l}0 \\
56\end{array}$ & $\begin{array}{l}0 \\
55\end{array}$ \\
\hline $\begin{array}{l}\underset{6}{\sigma} \\
0 \\
6\end{array}$ & $\stackrel{8}{8}$ & $\frac{\stackrel{\circ}{\hat{~}}}{\hat{a}}$ & $\stackrel{\circ}{\circ}$ & $\begin{array}{l}\stackrel{0}{0} \\
\text { ă }\end{array}$ & $\stackrel{\circ}{\circ}$ & $\stackrel{8}{8}$ & $\stackrel{\stackrel{\circ}{+}}{\stackrel{2}{r}}$ & $\begin{array}{l}\delta_{0}^{0} \\
\infty \\
\infty\end{array}$ & $\stackrel{8}{\varrho}$ & $\stackrel{8}{\circ}$ & & & & \\
\hline
\end{tabular}

$\alpha$ alpha haemolysis, $\beta$ beta haemolysis, + positive - negative

\section{Occurrence of $B$. cereus isolates in food samples}

The occurrence of $B$. cereus in the retailed food sample collected from the four geographical divisions in the state is presented on Table 4.The highest occurrence of $B$. cereus was from food samples from Ijebu while the least was from Egba division.

Table 4 Occurrence of $B$. cereus in some retailed food sample

\begin{tabular}{lccccc}
\hline $\begin{array}{l}\text { Location/ } \\
\text { Food code }\end{array}$ & $\begin{array}{c}\text { No of } \\
\text { isolates }\end{array}$ & Egba & Ijebu & Remo & Yewa \\
\hline WR & 33 & 8 & 7 & 8 & 10 \\
JR & 4 & 1 & 2 & 1 & - \\
FR & 6 & 2 & 2 & 1 & 1 \\
SG & 34 & 8 & 9 & 7 & 10 \\
MP & 10 & 2 & 3 & 2 & 3 \\
MT & 18 & 4 & 6 & 3 & 5 \\
SW & 27 & 4 & 8 & 8 & 7 \\
PN & 8 & 2 & 2 & 1 & 3 \\
PM & 7 & 1 & 3 & 2 & 1 \\
TT & 16 & 4 & 5 & 4 & 3 \\
GP & 8 & 2 & 1 & 4 & 1 \\
RB & 25 & 7 & 5 & 6 & 7 \\
CR & 5 & 1 & 1 & 2 & 1 \\
CB & 10 & 3 & 3 & 3 & 1 \\
SP & 10 & 3 & 1 & 4 & 2 \\
& 221 & 52 & 58 & 56 & 55 \\
& & $(23.5 \%)$ & $(26.2 \%)$ & $(25.3 \%)$ & $(24.0 \%)$ \\
\hline WR - White Rice & JR - Jollof Rice & FR - Fried Rice & TT -Smoked Titus & GP - Green Pea & SG-Spaghetti
\end{tabular}

- Runner Bean $\quad$ MP-Meat pie CR-Carrot $\quad$ MT- Meat $\quad$ CB - Cabbage $\quad$ SW-Smoked African chad $\quad$ SP-Sweet

Pepper PN-Smoked blue whiting PM-Smoked hide

\section{Antimicrobial Susceptibility Test}

All the isolates were resistant to penicillin and ampicillin while $99.5 \%$ were resistant to amoxicillin-clavulanic acid. They were all susceptible to gentamicin (Table 5). The resistance pattern of the B. cereus isolates from the retailed food is presented on Table 6 . Some of the isolates were resistant to more than one antibiotic. Resistance to penicillin, ampicillin and amoxicillin-clavulanic acid was displayed by $7.7 \%$ of the isolates, $22.6 \%$ showed resistance to penicillin, amoxicillin-clavulanic acid, erythromycin and ampicillin while $5.4 \%$ were resistant to all the antibiotics except gentamicin.

$\underline{\text { Table } 5 \text { Antibiotic Resistance of } B \text {. cereus from each location }}$

\begin{tabular}{|c|c|c|c|c|c|c|c|c|c|}
\hline 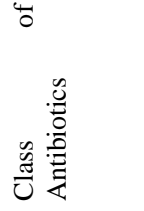 & $\begin{array}{c}\Xi \\
\frac{\Xi}{0} \\
\frac{\mathbb{E}}{\dot{1}}\end{array}$ & 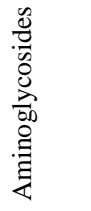 & $\begin{array}{l}\Xi \\
\frac{\Xi}{0} \\
\frac{d}{1} \\
\infty\end{array}$ & 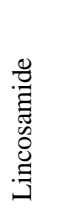 & $\begin{array}{l}\frac{0}{0} \\
: \frac{0}{0} \\
\stackrel{0}{0} \\
\sum\end{array}$ & $\frac{\mathscr{0}}{\frac{0}{0}}$ & 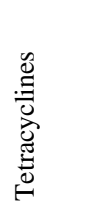 & 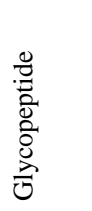 & 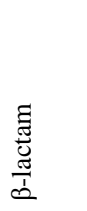 \\
\hline $\begin{array}{l}\text { Antibiotics/ } \\
\text { Location }\end{array}$ & $\begin{array}{l}\text { PEN } \\
(10 I U)\end{array}$ & $\begin{array}{l}\text { GMN } \\
(10 \mu \mathrm{g}) \\
\end{array}$ & $\begin{array}{l}\text { AMC } \\
(30 \mu \mathrm{g})\end{array}$ & $\begin{array}{l}\text { CMN } \\
(2 \mu \mathrm{g})\end{array}$ & $\begin{array}{l}\text { ERY } \\
(15 \mu \mathrm{g})\end{array}$ & $\begin{array}{l}\text { CIP } \\
(5 \mu \mathrm{g}) \\
\end{array}$ & $\begin{array}{l}\text { TET } \\
(30 \mu \mathrm{g}) \\
\end{array}$ & $\begin{array}{l}\text { VAN } \\
(30 \mu \mathrm{g}) \\
\end{array}$ & $\begin{array}{l}\text { AMP } \\
(10 \mu \mathrm{g})\end{array}$ \\
\hline EGBA & 52 & 0 & 52 & 23 & 33 & 19 & 17 & 25 & 52 \\
\hline IJEBU & 58 & 0 & 57 & 23 & 37 & 15 & 18 & 16 & 58 \\
\hline REMO & 56 & 0 & 56 & 25 & 45 & 15 & 19 & 24 & 56 \\
\hline YEWA & 55 & 0 & 55 & 19 & 33 & 17 & 13 & 21 & 55 \\
\hline TOTAL & 221 & 0 & 220 & 90 & 148 & 66 & 69 & 86 & 221 \\
\hline$(\%)$ & $100 \%$ & $0 \%$ & $99.5 \%$ & $40.7 \%$ & $67 \%$ & $29.9 \%$ & $30.3 \%$ & $38.9 \%$ & $100 \%$ \\
\hline
\end{tabular}

I - $\beta$-Lactam, II - Aminoglycosides, III - Lincosamide, IV - Macrolides, V - Quinolones, VI - Tetracycline, VII - Glycopeptides 
Table 6 Resistance pattern of B. cereus isolates from retailed foods

\begin{tabular}{|c|c|c|c|}
\hline Resistance pattern & Antibiotic class & $\begin{array}{c}\text { No of } \\
\text { isolates }\end{array}$ & $\begin{array}{c}\text { Resistance } \\
(\%)\end{array}$ \\
\hline PEN, AMC, AMP & I & 17 & 7.7 \\
\hline PEN, AMC, ERY, AMP & I,IV & 50 & 22.6 \\
\hline PEN, AMC, CIP, AMP & $\mathrm{I}, \mathrm{V}$ & 7 & 3.2 \\
\hline PEN, AMC, TET, AMP & $\mathrm{I}, \mathrm{VI}$ & 8 & 3.6 \\
\hline PEN, AMC, VAN, AMP & I,VII & 4 & 1.8 \\
\hline PEN, AMC, CMN, AMP & I,III & 11 & 5.0 \\
\hline $\begin{array}{l}\text { PEN, AMC, ERY, VAN, } \\
\text { AMP }\end{array}$ & $\mathrm{I}, \mathrm{IV}, \mathrm{VII}$ & 9 & 4.1 \\
\hline $\begin{array}{l}\text { PEN, AMC, CMN, } \\
\text { ERY, AMP }\end{array}$ & I,III,IV & 14 & 6.3 \\
\hline $\begin{array}{l}\text { PEN, AMC, CMN, } \\
\text { VAN, AMP }\end{array}$ & I,III,VII & 5 & 2.3 \\
\hline $\begin{array}{l}\text { PEN, AMC, CIP, TET, } \\
\text { AMP }\end{array}$ & $\mathrm{I}, \mathrm{V}, \mathrm{VI}$ & 5 & 2.3 \\
\hline $\begin{array}{l}\text { PEN, AMC, ERY, CIP, } \\
\text { AMP }\end{array}$ & $\mathrm{I}, \mathrm{IV}, \mathrm{V}$ & 4 & 1.8 \\
\hline $\begin{array}{l}\text { PEN, AMC, TET, VAN, } \\
\text { AMP }\end{array}$ & I,VI,VII & 1 & 0.5 \\
\hline $\begin{array}{l}\text { PEN, AMC, ERY, TET, } \\
\text { AMP }\end{array}$ & $\mathrm{I}, \mathrm{IV}, \mathrm{VI}$ & 3 & 1.4 \\
\hline $\begin{array}{l}\text { PEN, AMC, CMN, } \\
\text { ERY, AMP }\end{array}$ & I,III,IV & 1 & 0.5 \\
\hline $\begin{array}{l}\text { PEN, CMN, TET, VAN, } \\
\text { AMP }\end{array}$ & I,III,VI,VII & 1 & 0.5 \\
\hline $\begin{array}{l}\text { PEN, AMC, CMN, TET, } \\
\text { AMP }\end{array}$ & I,III,VI & 1 & 0.5 \\
\hline $\begin{array}{l}\text { PEN, AMC, CMN, CIP, } \\
\text { AMP }\end{array}$ & I,III,V & 2 & 0.9 \\
\hline $\begin{array}{l}\text { PEN, AMC, CMN, } \\
\text { ERY, TET, AMP }\end{array}$ & I,III,IV,VI & 1 & 0.5 \\
\hline $\begin{array}{l}\text { PEN, AMC, CMN, CIP, } \\
\text { TET, AMP }\end{array}$ & I,III,V,VI & 1 & 0.5 \\
\hline $\begin{array}{l}\text { PEN, AMC, ERY, CIP, } \\
\text { VAN, AMP }\end{array}$ & $\mathrm{I}, \mathrm{IV}, \mathrm{V}, \mathrm{VII}$ & 10 & 4.5 \\
\hline $\begin{array}{l}\text { PEN, AMC, CMN, } \\
\text { ERY, CIP, AMP }\end{array}$ & I,III,IV,V & 1 & 0.5 \\
\hline $\begin{array}{l}\text { PEN, AMC, CMN, TET, } \\
\text { VAN, AMP }\end{array}$ & I,III,VI,VII & 2 & 0.9 \\
\hline $\begin{array}{l}\text { PEN, AMC, ERY, CIP, } \\
\text { TET, AMP }\end{array}$ & $\mathrm{I}, \mathrm{IV}, \mathrm{V}, \mathrm{VI}$ & 3 & 1.4 \\
\hline $\begin{array}{l}\text { PEN, AMC, ERY, TET, } \\
\text { VAN, AMP }\end{array}$ & I,IV,VI,VII & 3 & 1.4 \\
\hline $\begin{array}{l}\text { PEN, AMC, CMN, } \\
\text { ERY, VAN, AMP }\end{array}$ & I,III,IV,VII & 12 & 5.4 \\
\hline $\begin{array}{l}\text { PEN, AMC, CMN, CIP, } \\
\text { VAN, AMP }\end{array}$ & I,III,V,VII & 3 & 1.4 \\
\hline $\begin{array}{l}\text { PEN, AMC, CMN, } \\
\text { ERY, TET, AMP }\end{array}$ & I,III,IV,VI & 3 & 1.4 \\
\hline $\begin{array}{l}\text { PEN, AMC, CIP, TET, } \\
\text { VAN, AMP }\end{array}$ & $\mathrm{I}, \mathrm{V}, \mathrm{VI}, \mathrm{VII}$ & 2 & 0.9 \\
\hline $\begin{array}{l}\text { PEN, AMC, ERY, CIP, } \\
\text { TET, VAN, AMP }\end{array}$ & $\mathrm{I}, \mathrm{IV}, \mathrm{V}, \mathrm{VI}, \mathrm{VII}$ & 5 & 2.3 \\
\hline $\begin{array}{l}\text { PEN, AMC, CMN, } \\
\text { ERY, TET, VAN, AMP }\end{array}$ & I,III,IV,VI,VII & 9 & 4.1 \\
\hline $\begin{array}{l}\text { PEN, AMC, CMN, } \\
\text { ERY, CIP, TET, AMP }\end{array}$ & $\mathrm{I}, \mathrm{III}, \mathrm{IV}, \mathrm{V}, \mathrm{VI}$ & 3 & 1.4 \\
\hline $\begin{array}{l}\text { PEN, AMC, CMN, CIP, } \\
\text { TET, VAN, AMP }\end{array}$ & I,III,V,VI,VII & 3 & 1.4 \\
\hline $\begin{array}{l}\text { PEN, AMC, CMN, } \\
\text { ERY, CIP, VAN, AMP } \\
\text { PEN AMC CMN }\end{array}$ & I,III,IV,V,VII & 5 & 2.3 \\
\hline $\begin{array}{l}\text { ERY, CIP, TET, VAN, } \\
\text { AMP }\end{array}$ & I,III,IV,V,VI,VII & 12 & 5.4 \\
\hline
\end{tabular}

- Ciprofloxacin TET-Tetracycline VAN-Vancomycin, AMP-Ampicillin

I - $\beta$-Lactam, II - Aminoglycosides, III - Lincosamide, IV - Macrolides, V - Quinolones, VI - Tetracycline, VII - Glycopeptides

Table 7 showed the percentage resistance for each food sample. All the isolates from each food sample showed $100 \%$ resistance to penicillin, ampicillin and amoxicillin-clavulanic acid except for isolates from PM which showed $85.7 \%$ resistance to amoxicillin-clavulanic acid. Isolates from CB and JR showed $100 \%$ resistance to erythromycin. PM isolates showed the highest resistance of $71.4 \%$ to tetracycline.
Table 7 Percentage (\%) resistance of $B$. cereus isolates in each food sample

\begin{tabular}{|c|c|c|c|c|c|c|c|c|c|}
\hline $\begin{array}{l}\text { Food } \\
\text { code }\end{array}$ & $\begin{array}{l}\text { No of } \\
\text { Isolates }\end{array}$ & $\begin{array}{l}\text { PEN } \\
(\%)\end{array}$ & $\begin{array}{l}\text { AMC } \\
(\%)\end{array}$ & $\begin{array}{l}\text { CMN } \\
(\%)\end{array}$ & $\begin{array}{l}\text { ERY } \\
(\%)\end{array}$ & $\begin{array}{l}\text { CIP } \\
(\%)\end{array}$ & $\begin{array}{l}\text { TET } \\
(\%)\end{array}$ & $\begin{array}{l}\text { VAN } \\
(\%)\end{array}$ & $\begin{array}{l}\text { AMP } \\
(\%)\end{array}$ \\
\hline WR & 33 & 100 & 100 & 33.3 & 81.8 & & & 57.6 & 100 \\
\hline SG & 34 & 100 & 100 & 41.2 & 76.4 & 48.5 & 24.2 & 47.1 & 100 \\
\hline SW & 27 & 100 & 100 & 29.6 & 74.1 & & & 29.6 & 100 \\
\hline MT & 18 & 100 & 100 & 61.1 & 22.2 & 29.4 & 35.3 & 38.9 & 100 \\
\hline $\mathrm{RB}$ & 25 & 100 & 100 & 32.0 & 32.0 & & & 48.0 & 100 \\
\hline TT & 16 & 100 & 100 & 50.0 & 68.8 & 33.3 & 29.6 & 37.5 & 100 \\
\hline $\mathrm{CB}$ & 10 & 100 & 100 & 30.0 & & & & 10.0 & 100 \\
\hline SP & 10 & 100 & 100 & 60.0 & 100.0 & 33.3 & 22.2 & 30.0 & 100 \\
\hline CR & 5 & 100 & 100 & 40.0 & 70.0 & & & 20.0 & 100 \\
\hline FR & 6 & 100 & 100 & 33.3 & 80.0 & 28.0 & 40.0 & 33.3 & 100 \\
\hline GP & 8 & 100 & 100 & 37.5 & 33.3 & & & 37.5 & 100 \\
\hline JR & 4 & 100 & 100 & 50.0 & 62.5 & 12.5 & 18.8 & 25.0 & 100 \\
\hline PM & 7 & 100 & 85.7 & 28.6 & & & & 42.9 & 100 \\
\hline PN & 8 & 100 & 100 & 25.0 & 100.0 & 10.0 & 10.0 & 25.0 & 100 \\
\hline \multirow[t]{10}{*}{ MP } & 10 & 100 & 100 & 40.0 & 85.7 & & & 40.0 & 100 \\
\hline & & & & & 62.5 & 60.0 & 40.0 & & \\
\hline & & & & & 80.0 & & & & \\
\hline & & & & & & 20.0 & 40.0 & & \\
\hline & & & & & & 0.0 & 0.0 & & \\
\hline & & & & & & 12.5 & 37.5 & & \\
\hline & & & & & & 0.0 & 0.0 & & \\
\hline & & & & & & 14.3 & 71.4 & & \\
\hline & & & & & & 37.5 & 25.0 & & \\
\hline & & & & & & 30.0 & 50.0 & & \\
\hline \multicolumn{2}{|c|}{ PEN - Penicillin } & Sentamicin & $\mathrm{AMC}-\mathrm{A}$ & xicillin-cle & anic acid & $\mathrm{CMN}-\mathrm{C}$ & idamycin & ERY- Ery & mycin \\
\hline \multicolumn{10}{|c|}{ CIP - Ciprofloxaci } \\
\hline - Fried R & $\mathrm{TT}-\mathrm{Smo}$ & Titus GP & -Green Pea & SG - Spag & & & Runner B & MP - & eat pie \\
\hline
\end{tabular}

\section{DISCUSSION}

Six hundred (600) different food samples were analysed for the presence of Bacillus cereus. The microbial load in the retailed food sample was high. Fang et al. (2003) also reported B. cereus load in $18^{\circ} \mathrm{C}$ ready-to-eat food products in the range of $10^{4}$ to $\geq 10^{5} \mathrm{cfu} / \mathrm{g}$. According to Gilbert et al. (2000) B. cereus count in food greater than $10^{4}-<10^{5}$ is unsatisfactory while $>10^{5}$ is unacceptable. There were significant differences in the microbial load of the food samples FR, $\mathrm{SW}, \mathrm{PN}, \mathrm{SP}, \mathrm{CB}$ and CR from the different locations at $\mathrm{p} \leq 0.05$.

Two hundred and twenty one (221) B. cereus isolates were recovered from the retailed foods analyzed. Ninety three point two (93.2\%) formed $\beta$ - haemolysis on sheep blood agar. Six strains were $\alpha$ haemolytic and nine were nonhaemolytic. This is in line with the work of Chaves et al., (2011) who reported $81.4 \%$ of their B. cereus to be $\beta$ - haemolytic, six were non haemolytic while seven were $\alpha$-haemolytic. Chon et al., (2012) reported that $89 \%$ of their strains were haemolytic. Eighty five point one $(86.0 \%)$ and seventy seven point four (77.4\%) of the strains hydrolysed skim milk, and starch agar. Chon et al. (2012) reported that $84 \%$ and $89 \%$ each of their strains hydrolysed lecithinase and starch. They all grew very well at $42^{\circ} \mathrm{C}$. This is in line with the findings of Lindbäck and Granum (2006) who reported that B. cereus grows fast at $42^{\circ} \mathrm{C}$. Detection of $B$. cereus in retailed foods in this research is in line with the work of Desai and Varadaraj (2009); Al-Abri et al. (2011); Naranjo et al. (2011); Martinelli et al. (2013); Lopez et al. (2015).

The rate of occurrence of $B$. cereus from the retailed food samples showed that samples from Ijebu division had the highest occurrence while Egba had the least occurrence. Sixteen percent $(16 \%)$ of $B$. cereus isolates were recovered from spaghetti. This may be as a result of poor hygiene practised by the food handlers. The food handlers put spaghetti in small transparent plastics buckets instead of hot food containers that can keep them hot. Also, most of them use their bare hands to support the spaghetti when serving it with fork to the consumers. These same hands were used to receive money and handle their food utensils. These can cause cross contamination from the money to the food because currency notes are known to harbour pathogenic microorganisms including Bacillus species (Uneke and Ogbu, 2007; Awe et al., 2010; Orukotan and Yabaya, 2011).

All the $B$. cereus strains were $100 \%$ sensitive to gentamicin. This compared well with the works of Chaves et al. (2011); Chon et al. (2012); Mohammed et al. (2012) and Organji et al. (2015) who reported $100 \%$ sensitivity to gentamicin by $B$. cereus strains recovered from food. Also, $62 \%$ of the strains in this study were sensitive to vancomycin, while $38 \%$ were resistant to the drug. Ankolekar et al. (2009) reported that $44 \%$ of the isolates were resistant to vancomycin, $56 \%$ were intermediate strains and none was sensitive to the drug while Organji $\boldsymbol{e t}$ al. 
(2015) reported $100 \%$ sensitivity to vancomycin. WR strains showed the highest resistance of $57.6 \%$ to the drug.

All the B. cereus strains were $100 \%$ resistant to penicillin and ampicillin while 99.5\% were resistant to amoxicillin-clavulanic acid. This conforms to the work of Park et al. (2009), Chon et al. (2012) and Savic et al. (2016) who reported that their strains displayed $100 \%$ resistance to ampicillin and penicillin. It was reported by Arslan et al. (2014) that $B$. cereus strains were resistant to ampicillin and penicillin $\mathrm{G}$ with the equal rate of $89.7 \%$ while $27.6 \%$ and $13.8 \%$ showed resistance and intermediate resistance respectively to amoxicillin-clavulanic acid. This is in contrast with the result in this work where higher percentage $(99.5 \%$ ) of the strains was resistant to amoxicillin-clavulanic acid. Penicillin, ampicillin and amoxicillin-clavulanic acid are $\beta$ - lactam antibiotics and resistance to these drugs is as a result of synthesis of $\beta$ - lactamase by $B$. cereus.

Sensitivity to ciprofloxacin was $70.1 \%, 29.0 \%$ showed intermediate resistance while $0.9 \%$ was completely resistant to the antibiotic. Jawad et al. (2016) reported that $42 \%$ of B. cereus strains from fried rice showed resistant to the antibiotic. This result contradicts the work of Luna et al. (2007); Chon et al. (2012); Savic et al. (2016) who reported that B. cereus isolates were $100 \%$ sensitive to ciprofloxacin. SP isolates showed $48.5 \%$ resistance to the drug. Resistance to clindamycin was $9.1 \%$; intermediate resistance was $30.7 \%$ while $60.2 \%$ were sensitive to the drug. This is in contrast to the work of Luna $\boldsymbol{e t}$ al. (2007) and Park et al. (2009) who reported that $15 \%$ and $72 \%$ of B. cereus strains were resistant to clindamycin. Likewise, Arslan et al. (2014) reported 6.9\% resistant to the drug while Chaves et al. (2011) recorded ten intermediate strains among B. cereus strains. However, some authors like Ankolekar et al. (2009), Chon et al. (2012), Organji et al. (2015) reported $100 \%$ sensitivity to the drug by B. cereus. MT strains showed the highest resistance of $61.1 \%$ to clindamycin.

Comparatively, the B. cereus strains in this research were highly resistance to erythromycin. Isolates from $\mathrm{CB}$ and JR demonstrated the highest resistance of $100 \%$ to erythromycin. Resistance to erythromycin was 38\%, Intermediate resistance was $29 \%$ while $33 \%$ were sensitive to the drug. However, the result of our study is supported by the work of Jawad et al. (2016) who reported that $42 \%$ of strains from fried rice were resistant to the drug. Other workers like Park et al. (2009), Chon et al. (2012) and Arslan et al. (2014) had reported 82\%, 93.1\% and $97 \%$ sensitivity to the drug respectively. Although, Ankolekar et al. (2009) reported that $58 \%$ of $B$. cereus strains from rice were resistant to erythromycin Authors like Organji et al. (2015) and Savic et al. (2016) reported 100\% sensitivity to the drug. A higher sensitivity to tetracycline (69\%) was obtained in this study compared to 54.8\% and 54\% sensitivity reported by Mohammed et al. (2012) and Chon et al. (2012) respectively. Ankolekar et al. (2009) reported that 98\% of their B. cereus strains were resistant to tetracycline. Park et al. (2009) and Jawad et al. (2016) stated that $85 \%$ and $86 \%$ of their isolates were sensitive while Savic et al. (2016) reported $100 \%$ sensitivity to the drug. Resistance to the drug was displayed by $71.4 \%$ of PM strains while the least resistance was $10.0 \%$ by $\mathrm{CB}$ isolates. PM is animal hide and the high resistance may be as a result of misuse of antibiotics.

More than $50 \%$ of the isolates displayed multiple drug resistances i.e. resistance to three or more antimicrobial classes. The highest resistance was displayed by fourteen strains which were resistant to three classes of antibiotics followed by twelve strains that were resistant to all the antibiotics except gentamicin.

\section{CONCLUSION}

This research work has revealed that $B$. cereus contaminated some retailed foods sold in Ogun State especially the samples from Ijebu division and are multiresistant to common antibiotics. Therefore extra caution should be taken when handling foods to avoid contamination of the food from the handler and the environment.

Acknowledgement: The authors appreciate the financial support provided by the University management through the Tertiary Education Tax Fund (TETFUND) and the Microbial Biotechnology Laboratory, NWU, South Africa for hosting the first author.

\section{REFERENCES}

Al-Abri, S.S., Al-Jardania, A.K., Al-Hosnia, M.S., Kurupb, P.J., Al-Busaidi, S., \& Beechingd, N.J. (2011). A hospital

acquired outbreak of Bacillus cereus gastroenteritis, Oman. Journal of Infection and Public Health, 4,180-186.http://dx. doi.org/10.1016/j.jiph.2011.05.003.

Angelidis, A., Chronis, G., Papageorgiou, D., Kazakis, I., Arsenoglou, K., \& Stathopoulos, G. (2006). Non-lactic acid, contaminating microbial flora in readyto-eat foods: A potential food-quality index. Food Microbiology, 23,95-100. http://dx.doi.org/10.1016/i.fm.2005.01.015.

Ankolekar, C., Rahmati, T., \& Labbé, R.G. (2009). Detection of toxigenic Bacillus cereus and Bacillus thuringiensis spores in U.S. rice. International Journal of Food Microbiology, 128(3),460-466. http://dx.doi.org/10.1016/j.ijfoodmicro.2008.10.006.
ARSLAN, S., EYI, A., \& KÜÇÜKSARI, R. (2014). Toxigenic genes, spoilage potential, and antimicrobial resistance of Bacillus cereus group strains from ice cream. Anaerobe, 25,42-46. http://dx.doi.org/10.1016/j.anaerobe.2013.11.006. Awe, S., Eniola, K.I.T., Ojo, F.T., \& Sani, A. (2010). Bacteriological quality of some Nigerian currencies in circulation. African Journal of Microbiology Research, 4(21), 2231- 2234.

Bauer, A.W., Kirby, W.M., Sherris, J.C., \& Turck, M. (1966). Antibiotic susceptibiity testing by a standardized single disk method. America Journal of Clinical Pathology, 45(4), 493-496.

Chaves, J.Q., Pires, E.S., \& Vivoni, A.M. (2011). Genetic diversity, antimicrobial resistance and toxigenic profiles of Bacillus cereus isolated from food in Brazil over three decades. International Journal of Food Microbiology, 147(1), 12-16. http://dx.doi.org/10.1016/j.ijfoodmicro.2011.02.029.

Cheesebrough, M. (2000). District Laboratory Practice in Tropical countries Part 2. London: Cambridge University Press.

Chon, J.W., Kim, J.H., Lee, S.J., Hyeon, J.Y., \& Seo, K.H. (2012). Toxin profile, antibiotic resistance, and phenotypic and molecular characterization of Bacillus cereus in Sunsik. Food Microbiology, 32, 217-222. http://dx.doi.org/10.1016/j.fm.2012.06.003.

CLSI (2013). Performance standards for antimicrobial disc susceptibility tests; Twenty third informational supplement M100 - S23 (M02- A11). Clinical and Laboratory Standards Institutes, Wayne, Pennsylvania.

Das, S., Surendran, P., \& Thampuran, N. (2009). PCR-based detection of enterotoxigenic isolates of Bacillus cereus from tropical seafood. Indian Journal of Medical Research, 129, 316-320.

De Jonghe, V., Coorevits, A., Vandroemme, J., Heyrman, J., Herman, L., De Vos, P., \& Heyndrickx, M. (2008). Intraspecific genotypic diversity of Bacillus species from raw milk. International Dairy Journal,18, 496-505. http://dx.doi.org/10.1016/j.idairyj.2007.11.007

Desai, S.V., \& Varadaraj, M.C. (2009). Prevalence of toxigenic traits in native food isolates of Bacillus cereus in the city of Mysore, Southern India. Journal of Microbiology and Antimicrobials, 1, 027-034.

Dierick, K., Van Coillie, E., Swiecicka, I., Meyfroidt, G., Devlieger, H., Meulemans, A., Hoedemaekers, G., Fourie, L., Heyndrickx, M., \& Mahillon, J. (2005). Fatal family outbreak of Bacillus cereus-associated food poisoning. Journal of Clinical Microbiology, 43(8), 4277-4279. http://dx.doi.org/10.1128/JCM.43.8.4277-4279.2005.

Fang, T.J., Wei, Q.K., Liao, C.W., Hung, M.J., \& Wang, T.H. (2003) Microbiological quality of $18^{\circ} \mathrm{C}$ ready-to-eat food products sold in Taiwan International Journal of Food Microbiology, 80(3), 241-250.

Ghelardi, E., Celandroni, F., Salvetti, S., Barsotti, C., Baggiani, A., \& Senesi, S (2002). Identification and characterization of toxigenic Bacillus cereus isolates responsible for two food poisoning outbreaks. FEMS Microbiology Letters, 208, 129-134.

Gilbert, R.J., De Louvois, J., Donovan, T., Little, C., Nye, K., Ribeiro, C.D., Richards, J., Roberts, D., \& Bolton, F.J. (2000). Guidelines for the microbiological quality of some ready-to-eat foods sampled at the point of sale. Commun. Dis. Public Health, 3, 163-167.

Gueven, K., Mutlu, M.B., \& Avci, O. (2006). Incidence and characterization of Bacillus cereus in meat and meat products consumed in Turkey. Journal of Food Safety, 26,30-40 http://dx.doi.org/10.1111/j.1745-4565.2005.00031.x

IBM Corporation (2011). IBM SPSS statistics for Windows version 20.0 Armonk, NY:IBM Corp.

Iurlina, M.O., Saiz, A.I., Fuselli, S.R., \& Fritz, R. (2006). Prevalence of Bacillus spp. in different food products collected in Argentina. LWT - Food Science and Technology, 39,105-110. http://dx.doi.org/10.1111/j.1750-3841.2010.01566.x.

Jawad, N., Abd Mutalib, S., \& Abdullah, A. (2016). Antimicrobial resistance pattern of Bacillus cereus strains isolated from fried rice samples. International Journal of ChemTech Research, 9(1), 160-167.

Lapidus, A., Goltsman, E., Auger, S., Galleron, N., Ségurens, B., Dossat, C., Land, M., Broussolle, V., Brillard, J., Guinebretiere, M.H., Sanchis, V., NguyenThe, C., Leredus, D., Richardson, P., Wincker, P., Weissenbach, J., Ehrlich, S.D. \& Sorokin, A. (2008). Extending the Bacillus cereus group genomics to putative food-borne pathogens of different toxicity. Chemico-Biological Interactions, 171(2), 236-249. http://dx.doi.org/10.1016/j.cbi.2007.03.003.

Lindbäck, T., \& Granum, P.E. (2006). Detection and Purification of Bacillus cereus Enterotoxins. In C.C. Adley (Ed.), Food-Borne Pathogens: Methods and Protocols (pp. 15-26). New Jersey: Humana Press Inc.

Logan, N.L., \& De Vos, P. (2009). Genus I. Bacillus. In P. de Vos, G.M. Garrity, D. Jones, N.R. Krieg, W. Ludwig, F.A. Rainey et al. (Eds.), Bergey's Manual of Systematic Bacteriology (pp. 21-128). New York: Springer.

López, A.C., Minnaard, J., Pérez, P.F., \& Alippi, A.M. (2015). A case of intoxication due to a highly cytotoxic Bacillus cereus strain isolated from cooked $\begin{array}{llll}\text { chicken. } & \text { Food } & \text { Microbiology, } & \text { 465-199. }\end{array}$ ,http://dx.doi.org/10.1016/j.fm.2014.08.005.

Luna, V.A., King, D.S., Gulledg, E, J., Cannons, A.C., Amiso, P.T., \& Cattani, J. (2007). Susceptibility of bacillus anthracis, Bacillus cereus, Bacillus mycoides, Bacillus pseudomycoides and B. thuringensis to 24 antimicrobials using sensititre 
automated microbroth dilution and Etest agar gradient diffusion methods. Journal of Antimicrobial Chemotherapy, 60, 555-5670.

Martinelli, D., Fortunato, F., Tafuri, S., Cozza, V., Chironna, M., Germinario, C., Pedalino, B., \& Prato, R. (2013). Lessons from a birthday party: a Bacillus cereus outbreak, Bari, Italy, January 2012. Ann $1^{\text {st }}$ Super Sanita, 49 (4), 391 -394 http://dx.doi.org/10.4415/ann $13 \quad 04 \quad 12$

Mohammed, K.H., Basil, A.A., \& Ban, M.S. (2012). Molecular detection of Enterotoxin $(C y t K)$ Gene and Antimicrobial susceptibility of B. cereus isolates from milk and milk products. Basrah Journal of Veterinary Research, 11(1), 164 173

Naranjo, M., Denayer, S., Bottledorm, N., Delbrassinne, L., Veys, J., Waegenaere, J., Sirtaine, N., Driesen, R.B., Sipido, K.R., Mahillon, J., \& Dierick, K. (2011). Sudden Death of a Young Adult Associated with Bacillus cereus Food Poisoning. Journal of Clinical Microbiology, 49(12), 4379-4381. http://dx.doi.org/10.1128/JCM.05129-11.

Organji, S.R., Abulreesh, H.H., Elbanna, K., Osman, G.E.H., \& Khider, M (2015). Occurrence and characterization of toxigenic Bacillus cereus in food and infant feces. Asian Pacific Journal of Tropical Biomedicine, 5, 515-520. http://dx.doi.org/10.1016/j.apjtb.2015.04.004.

Orukotan, A.A., \& Yabaya, A. (2011). Microbial Contamination of Naira Notes in Circulation within Kaduna Metropolis. Journal of Medical and Applied Biosciences, 2, 20-27.

Park, Y.B., Kim, J.B., Shin, S.G., Kim, J.C., Cho, S.H, Lee, B.K., Ahn, J., Kim, J.M., \& Oh, D.H. (2009). Prevalence, Genetic Diversity, and Antibiotic Susceptibility of Bacillus cereus Strains from Rice and Cereals collected in Korea. Journal of Food Protection, 72(3), 612-617.

Rajkovic, A., Kljajic, M., Smigic, N., Devlieghere, F., \& Uyttendaele, M. (2013). Toxin producing Bacillus cereus persist in ready-to-reheat spaghetti Bolognese mainly in vegetative state. International Journal of Food Microbiology, 167 236-243. http://dx.doi.org/10.1016/j.ijfoodmicro.2013.09.001.

Samapundo, S., Heyndrickx, M., Xhaferi, R., \& Devlieghere, F. (2011) Incidence, diversity and toxin gene characteristics of Bacillus cereus group strains isolated from food products marketed in Belgium. International Journal of Food Microbiology, 150(1), 34-41. http://dx.doi.org/10.1016/j.ijfoodmicro.2011.07.013

Savic, D., Miljkovic-Selimovic, B., Lepsanovic, Z., Tambur, Z., Konstantinovic S., Stankovic, N., \& Ristanovic, E. (2016). Antimicrobial Susceptibility and $\beta$ lactamase production in B. cereus isolatesfrom stool of patients, food and environment samples. Vojnosanit Preg, 73(10), 904-909. http://dx.doi.org/10.2298/VSP150415134S.

Tallent, S.M., Jeffery Rhodehamel E.J., Harmon, S.M., \& Bennett, R.W. (2001) Bacillus cereus. Bacteriological

Analytical Manual. Chapter 14.

Uneke, C.J., \& Ogbu, O. (2007). Potential for parasite and bacterial transmission by paper currency in Nigeria. Journal of Environmental Health, 69, 54- 60.

Valero, M., Hernández-Herrero, L.A., Fernández, P.S., \& Salmerón, M.C. (2002). Characterization of Bacillus cereus isolates from fresh vegetables and refrigerated minimally processed foods by biochemical and physiological tests. Food Microbiology, 19, 491-499. http://dx.doi.org/10.1006/yfmic.507.

Valero, M., Hernández-Herrero, L.A., \& Giner, M.J. (2007). Survival, isolation and characterization of a psychrotrophic Bacillus cereus strain from a mayonnaise-based ready-to-eat vegetable salad. Food Microbiology, 24, 671-677. http://dx.doi.org/10.1016/j.fm.2007.04.005

Zhou, G., Bester, K., Liao, B., Yang, Z., Jiang, R., \& Hendriksen, N.B. (2014) Characterization of Three Bacillus Strains Involved in a Major Outbreak of Food Poisoning After Consumption of Fermented Black Beans (Douchi) in Yunan, China. Food Borne Pathogens and Disease, 11(10), 69-774. http://dx.doi.org/ 10.1089/fpd.2014.1768 